\title{
A Computational Method for Two-Point Boundary Value Problems of Fourth-Order Mixed Integrodifferential Equations
}

\author{
Mohammed Al-Smadi, ${ }^{1}$ Omar Abu Arqub, ${ }^{2}$ and Shaher Momani ${ }^{3}$ \\ ${ }^{1}$ Department of Mathematics, Tafila Technical University, Tafila 66110, Jordan \\ ${ }^{2}$ Department of Mathematics, Al-Balqa Applied University, Salt 19117, Jordan \\ ${ }^{3}$ Department of Mathematics, University of Jordan, Amman 11942, Jordan
}

Correspondence should be addressed to Omar Abu Arqub; o.abuarqub@bau.edu.jo

Received 27 July 2012; Accepted 29 January 2013

Academic Editor: Hung Nguyen-Xuan

Copyright (C) 2013 Mohammed Al-Smadi et al. This is an open access article distributed under the Creative Commons Attribution License, which permits unrestricted use, distribution, and reproduction in any medium, provided the original work is properly cited.

In this paper, reproducing kernel Hilbert space method is applied to approximate the solution of two-point boundary value problems for fourth-order Fredholm-Volterra integrodifferential equations. The analytical solution was calculated in the form of convergent series in the space $W_{2}^{5}[a, b]$ with easily computable components. In the proposed method, the $n$-term approximation is obtained and is proved to converge to the analytical solution. Meanwhile, the error of the approximate solution is monotone decreasing in the sense of the norm of $W_{2}^{5}[a, b]$. The proposed technique is applied to several examples to illustrate the accuracy, efficiency, and applicability of the method.

\section{Introduction}

Boundary value problems (BVPs) of fourth-order integrodifferential equations (IDEs) which are a combination of differential and integral equations arise very frequently in many branches of applied mathematics and physics such as fluid dynamics, biological models, chemical kinetics, biomechanics, electromagnetic, elasticity, electrodynamics, heat and mass transfer, and oscillation theory [1-4]. If the BVPs for fourth-order Fredholm-Volterra IDEs cannot be solved analytically, which is the usual case, then recourse must be made to numerical and approximate methods.

This paper discusses and investigates the analytical approximate solution using reproducing kernel Hilbert space (RKHS) method for fourth-order Fredholm-Volterra IDE which is as follows:

$$
\begin{aligned}
u^{(4)}(x)= & f(x)+N(x, u(x))+\int_{a}^{b} h_{1}(x, t) G_{1}(u(t)) d t \\
& +\int_{a}^{x} h_{2}(x, t) G_{2}(u(t)) d t, \quad a \leq t, \quad x \leq b,
\end{aligned}
$$

subject to the boundary conditions

$$
\begin{array}{ll}
u(a)=\alpha_{0}, & u^{\prime}(a)=\alpha_{1}, \\
u(b)=\beta_{0}, & u^{\prime}(b)=\beta_{1},
\end{array}
$$

where $\alpha_{i}, \beta_{i}, i=0,1$ are real finite constants, $u \in W_{2}^{5}[a, b]$ is an unknown function to be determined, $f \in W_{2}^{1}[a, b]$, $h_{1}(x, t), h_{2}(x, t)$ are continuous functions on $[a, b]^{2}, G_{1}(w)$, $G_{2}(y), N(x, z)$ are continuous terms in $W_{2}^{1}[a, b]$ as $w=w(x)$, $y=y(x), z=z(x) \in W_{2}^{5}[a, b], a \leq x \leq b,-\infty<w, y, z<\infty$ and are depending on the problem discussed, and $W_{2}^{1}[a, b]$, $W_{2}^{5}[a, b]$ are two reproducing kernel spaces.

Recently, many authors have discussed the numerical solvability of BVPs for fourth-order Volterra IDEs which are special cases of (1) and (2). To mention a few, in [5], the authors have discussed the Adomian decomposition method for solving IDEs (1) and (2) when $N(x, u(x))=u(x)$, $G_{1}(u(t))=0$, and $G_{2}(u(t))=[u(t)]^{p}$, where $p \in \mathbb{N}$. The differential transform method has been applied to solve the same equations when $N(x, u(x))=u(x), G_{1}(u(t))=0$, and $G_{2}(u(t))=[u(t)]^{p}$, where $p \in \mathbb{N}$ as described in [6]. 
Furthermore, the homotopy perturbation method is carried out in [7] for the aforementioned IDEs in the case that $N(x, u(x))=u(x), G_{1}(u(t))=0$, and $G_{2}(u(t))=[u(t)]^{p}$, where $p \in \mathbb{N}$. Recently, the homotopy analysis method for solving (1) and (2) when $N(x, u(x))=u(x), G_{1}(u(t))=0$, and $G_{2}(u(t))=[u(t)]^{p}$, where $p \in \mathbb{N}$, is proposed in [8]. But on the other aspects as well, the numerical solvability of differential and integral equations of different type and order can be found in [9-15] and references therein.

Investigation about two-point BVPs for fourth-order Fredholm-Volterra IDEs is scarce. Actually, no one has given a method to get approximate solution for this type of equations in the literature. Also, none of previous studies propose a methodical way to solve these equations. Moreover, previous studies require more effort to achieve the results; they are not accurate but are usually developed for special types of (1) and (2). The new method is accurate, needs less effort to achieve the results, and is developed especially for nonlinear case. Meanwhile, the proposed technique has an advantage that it is possible to pick any point in the interval of integration, and the approximate solutions and all its derivatives up to order four will be applicable as well.

Reproducing kernel theory has important application in numerical analysis, differential equations, integral equations, probability and statistics, and so forth [16-18]. In the last years, extensive work has been done using RKHS method, which provides numerical approximations for linear and nonlinear equations. This method has been implemented in several operator, differential, integral, and integro-differential equations, such as nonlinear operator equations [19], nonlinear system of second-order BVPs [20], nonlinear fourth-order BVPs [21], nonlinear systems of initial value problems [22], nonlinear second-order singular BVPs [23], nonlinear partial differential equations [24], nonlinear Fredholm-Volterra integral equations [25], nonlinear fourthorder Volterra IDEs [26], nonlinear Fredholm-Volterra IDEs [27], and others.

The paper is organized in the following form. In the next section, two reproducing kernel spaces are described. In Section 3, a linear operator, a complete normal orthogonal system, and some essential results are introduced. Also, a method for the existence of solutions for (1) and (2) based on reproducing kernel space is described. In Section 4, we give an iterative method to solve (1) and (2) numerically in the space $W_{2}^{5}[a, b]$. Numerical examples are presented in Section 5. Section 6 ends this paper with a brief conclusion.

\section{Two Reproducing Kernel Spaces}

In this section, two reproducing kernels needed are constructed in order to solve (1) and (2) using RKHS method. Before the construction, we utilize the reproducing kernel concept. Throughout this paper, $\mathbb{C}$ is the set of complex numbers, $L^{2}[a, b]=\left\{u \mid \int_{a}^{b} u^{2}(x) d x<\infty\right\}$, and $l^{2}=\{A \mid$ $\left.\sum_{i=1}^{\infty}\left(A_{i}\right)^{2}<\infty\right\}$.

An abstract set is supposed to have elements, each of which has no structure, and is itself supposed to have no internal structure, except that the elements can be distinguished as equal or unequal, and to have no external structure except for the number of elements.

Definition 1 (see [23]). Let $E$ be a nonempty abstract set. A function $K: E \times E \rightarrow \mathbb{C}$ is a reproducing kernel of the Hilbert space $H$ if

(1) for each $x \in E, K(\cdot, x) \in H$;

(2) for each $x \in E$ and $\varphi \in H,\langle\varphi(\cdot), K(\cdot, x)\rangle=\varphi(x)$.

The second condition in Definition 1 is called "the reproducing property"; the value of the function $\varphi$ at the point $x$ is reproducing by the inner product of $\varphi(\cdot)$ with $K(\cdot, x)$. A Hilbert space which possesses a reproducing kernel is called an RKHS [23].

Next, we first construct the space $W_{2}^{5}[a, b]$ in which every function satisfies the boundary conditions (2) and then utilize the space $W_{2}^{1}[a, b]$.

Definition 2 (see [21]). $W_{2}^{5}[a, b]=\left\{u(x) \mid u^{(i)}, i=0,1,2,3,4\right.$ are absolutely continuous real-valued functions on $[a, b]$, $u^{(5)} \in L^{2}[a, b]$, and $\left.u(a)=u^{\prime}(a)=u(b)=u^{\prime}(b)=0\right\}$. The inner product and the norm in $W_{2}^{5}[a, b]$ are defined as

$$
\begin{aligned}
\langle u, v\rangle_{W_{2}^{5}}= & \sum_{i=0}^{2} u^{(i)}(a) v^{(i)}(a)+\sum_{i=0}^{1} u^{(i)}(b) v^{(i)}(b) \\
& +\int_{a}^{b} u^{(5)}(y) v^{(5)}(y) d y
\end{aligned}
$$

and $\|u\|_{W_{2}^{5}}=\sqrt{\langle u, u\rangle_{W_{2}^{5}}}$, respectively, where $u, v \in W_{2}^{5}[a, b]$.

Definition 3 (see [19]). $W_{2}^{1}[a, b]=\{u(x) \mid u$ is absolutely continuous real-valued function on $[a, b]$ and $\left.u^{\prime} \in L^{2}[a, b]\right\}$. The inner product and the norm in $W_{2}^{1}[a, b]$ are defined as $\langle u, v\rangle_{W_{2}^{1}}=\int_{a}^{b}\left(u^{\prime} v^{\prime}+u v\right) d y$ and $\|u\|_{W_{2}^{1}}=\sqrt{\langle u, u\rangle_{W_{2}^{1}}}$, respectively, where $u, v \in W_{2}^{1}[a, b]$.

Remark 4. In [19], the authors have proved that the space $W_{2}^{1}[a, b]$ is a complete reproducing kernel space and its reproducing kernel is

$$
\begin{aligned}
R_{x}(y)=\frac{1}{2 \sinh (b-a)}[ & \cosh (x+y-b-a) \\
& +\cosh (|x+y|-b+a)] .
\end{aligned}
$$

The Hilbert space $W_{2}^{5}[a, b]$ is called a reproducing kernel if for each fixed $x \in[a, b]$ and any $u(y) \in W_{2}^{5}[a, b]$, there exist $K(x, y) \in W_{2}^{5}[a, b]$ (simply $\left.K_{x}(y)\right)$ and $y \in[a, b]$ such that $\left\langle u(y), K_{x}(y)\right\rangle_{W_{2}^{5}}=u(x)$. The next theorem formulates the reproducing kernel function $K_{x}(y)$ on the space $W_{2}^{5}[a, b]$. 
Theorem 5. The Hilbert space $W_{2}^{5}[a, b]$ is a reproducing kernel and its reproducing kernel function $K_{x}(y)$ is given by

$$
K_{x}(y)= \begin{cases}\sum_{i=0}^{9} p_{i}(x) y^{i}, & y \leq x, \\ \sum_{i=0}^{9} q_{i}(x) y^{i}, & y>x,\end{cases}
$$

where $p_{i}(x)$ and $q_{i}(x)$ are unknown coefficients of $K_{x}(y)$.

Proof. Through several integrations by parts for (3), one can obtain $\left\langle u(y), K_{x}(y)\right\rangle_{W_{2}^{5}}=\sum_{i=0}^{2} u^{(i)}(a) K_{x}^{(i)}(a)+\sum_{i=0}^{1} u^{(i)}(b)$ $K_{x}^{(i)}(b)+\left.\sum_{i=0}^{4}(-1)^{4-i} u^{(i)}(y) K_{x}^{(9-i)}(y)\right|_{y=a} ^{y=b}-\int_{a}^{b} u(y) K_{x}^{(10)}(y) d y$. Since $K_{x}(y) \in W_{2}^{5}[a, b]$, it follows that $K_{x}^{(i)}(a)=K_{x}^{(i)}(b)=0$, $i=0,1$. Again, since $u \in W_{2}^{5}[a, b]$, one obtains $u^{(i)}(a)=$ $u^{(i)}(b)=0, i=0,1$. Thus, if $K_{x}^{(2)}(a)-K_{x}^{(7)}(a)=0$, $K_{x}^{(i)}(a)=0, i=5,6$, and $K_{x}^{(i)}(b)=0, i=5,6,7$, then $\left\langle u(y), K_{x}(y)\right\rangle_{W_{2}^{5}}=\int_{a}^{b} u(y)\left(-K_{x}^{(10)}(y)\right) d y$. Now, for each $x \in$ $[a, b]$, if $K_{x}(y)$ also satisfies the formula $-K_{x}^{(10)}(y)=\delta(x-y)$, where $\delta$ is the dirac-delta function, then $\left\langle u(y), K_{x}(y)\right\rangle_{W_{2}^{5}}=$ $u(x)$. Obviously, $K_{x}(y)$ is the reproducing kernel of the space $W_{2}^{5}[a, b]$.

Next, we give the expression of the reproducing kernel function $K_{x}(y)$. The characteristic equation of $-K_{x}^{(10)}(y)=$ $\delta(x-y)$ is $\lambda^{10}=0$, and their characteristic values are $\lambda=0$ with 10 multiple roots. So, let the kernel $K_{x}(y)$ be as defined in (5).

On the other hand, let $K_{x}(y)$ satisfy $K_{x}^{(m)}(x+0)=$ $K_{x}^{(m)}(x-0), m=0,1, \ldots, 8$. Integrating $-K_{x}^{(10)}(y)=\delta(x-y)$ from $x-\varepsilon$ to $x+\varepsilon$ with respect to $y$ and letting $\varepsilon \rightarrow 0$, we have the jump degree of $K_{x}^{(9)}(y)$ at $y=x$ given by $K_{x}^{(9)}(x-0)-K_{x}^{(9)}(x+0)=1$. Through the last descriptions, the unknown coefficients of (5) can be obtained. This completes the proof.

Remark 6. Without the loss of generality and by using Mathematica 7.0 software package, the two rules $K_{1 x}(y)=$ $\sum_{i=0}^{9} p_{i}(x) y^{i}$ and $K_{2 x}(y)=\sum_{i=0}^{9} q_{i}(x) y^{i}$ of the reproducing kernel function $K_{x}(y)$ in (5) are obtained at $a=0$ and $b=1$ in (1) and (2) and are given, respectively, as

$$
\begin{aligned}
K_{1 x}(y) & \frac{1}{362880}(x-1)^{2} y^{2} \\
& \quad \times\left(\begin{array}{c}
5 x^{3}(8-15 y) y+x^{6}(19-12 y) y+y^{7}+x y^{6}(-9+2 y) \\
+3 x^{5} y(-10+3 y)+x^{7} y(-4+3 y)+5 x^{4} y(1+6 y) \\
+3 x^{2}\left(30240-60480 y+30240 y^{2}+12 y^{5}-6 y^{6}+y^{7}\right)
\end{array}\right), \\
K_{2 x}(y) & \frac{1}{362880} x^{2}(y-1)^{2} \\
& \times\left(\begin{array}{c}
90720 y^{2}+36 x^{5} y^{2}-9 x^{6} y(1+2 y)+x^{7}\left(1+2 y+3 y^{2}\right) \\
+x y^{2}\left(-181440+40 y+5 y^{2}-30 y^{3}+19 y^{4}-4 y^{5}\right) \\
+3 x^{2} y^{2}\left(30240-25 y+10 y^{2}+3 y^{3}-4 y^{4}+y^{5}\right)
\end{array}\right) .
\end{aligned}
$$

The following corollary summarizes some important properties of the reproducing kernel function $K_{x}(y)$.

Corollary 7. The reproducing kernel function $K_{x}(y)$ is symmetric and unique, and $K_{x}(x) \geq 0$ for any fixed $x \in[a, b]$.

Proof. By the reproducing property, we have $K_{x}(y)=$ $\left\langle K_{x}(\xi), K_{y}(\xi)\right\rangle=\left\langle K_{y}(\xi), K_{x}(\xi)\right\rangle=K_{y}(x)$ for each $x$ and $y$. Now, let $K_{x}^{1}(y)$ and $K_{x}^{2}(y)$ be all the reproducing kernels of the space $W_{2}^{5}[a, b]$; then, $K_{x}^{1}(y)=\left\langle K_{x}^{1}(\xi), K_{y}^{2}(\xi)\right\rangle=$ $\left\langle K_{y}^{2}(\xi), K_{x}^{1}(\xi)\right\rangle=K_{y}^{2}(x)=K_{x}^{2}(y)$. Finally, we note that $K_{x}(x)=\left\langle K_{x}(\xi), K_{x}(\xi)\right\rangle=\left\|K_{x}(\xi)\right\|^{2} \geq 0$.

\section{Main Results and the Structure of Solution}

In this section, the representation of the analytical solution of (1) and (2) and the implementation method are given in the reproducing kernel space $W_{2}^{5}[a, b]$. After that, we construct an orthogonal function system of $W_{2}^{5}[a, b]$ based on the Gram-Schmidt orthogonalization process.

To do this, we define a differential operator $L$ as

$$
L: W_{2}^{5}[a, b] \longrightarrow W_{2}^{1}[a, b],
$$

such that

$$
L u(x)=u^{(4)}(x) .
$$

After homogenization of the boundary conditions (2), (1) and (2) can be converted into the equivalent form as follows:

$$
\begin{gathered}
\operatorname{Lu}(x)=F(x, u(x), T u(x), S u(x)), \quad a \leq x \leq b, \\
u(a)=0, \quad u^{\prime}(a)=0, \\
u(b)=0, \quad u^{\prime}(b)=0,
\end{gathered}
$$

such that $F(x, u(x), T u(x), S u(x))=f(x)+N(x, u(x))+$ $T u(x)+S u(x), T u(x)=\int_{a}^{b} h_{1}(x, t) G_{1}(u(t)) d t$, and $\operatorname{Su}(x)=\int_{a}^{x} h_{2}(x, t) G_{2}(u(t)) d t$, where $u(x) \in W_{2}^{5}[a, b]$ and $F(x, w, y, z) \in W_{2}^{1}[a, b]$ for $w=w(x), y=y(x), z=z(x) \epsilon$ $W_{2}^{5}[a, b], a \leq x \leq b,-\infty<w, y, z<\infty$. It is easy to show that $L$ is a bounded linear operator from $W_{2}^{5}[a, b]$ into $W_{2}^{1}[a, b]$.

Next, we construct an orthogonal function system of $W_{2}^{5}[a, b]$. Put $\varphi_{i}(x)=R_{x_{i}}(x)$ and $\psi_{i}(x)=L_{i}^{*} \varphi(x)$, where $\left\{x_{i}\right\}_{i=1}^{\infty}$ is dense on $[a, b]$ and $L^{*}$ is the adjoint operator of $L$. In terms of the properties of reproducing kernel function $R_{x}(y)$, one obtains $\left\langle u(x), \psi_{i}(x)\right\rangle_{W_{2}^{5}}=\left\langle u(x), L^{*} \varphi_{i}(x)\right\rangle_{W_{2}^{5}}=$ $\left\langle L u(x), \varphi_{i}(x)\right\rangle_{W_{2}^{1}}=L u\left(x_{i}\right), i=1,2, \ldots$

Remark 8. The orthonormal function system $\left\{\bar{\psi}_{i}(x)\right\}_{i=1}^{\infty}$ of the space $W_{2}^{5}[a, b]$ can be derived from Gram-Schmidt orthogonalization process of $\left\{\psi_{i}(x)\right\}_{i=1}^{\infty}$ as follows:

$$
\bar{\psi}_{i}(x)=\sum_{k=1}^{i} \beta_{i k} \psi_{k}(x)
$$


where $\beta_{i k}$ are orthogonalization coefficients and are given by the following subroutine:

$$
\begin{gathered}
\beta_{i j}=\frac{1}{\left\|\psi_{1}\right\|}, \quad \text { for } i=j=1, \\
\beta_{i j}=\frac{1}{d_{i k}}, \quad \text { for } i=j \neq 1, \\
\beta_{i j}=-\frac{1}{d_{i k}} \sum_{k=j}^{i-1} c_{i k} \beta_{k j}, \quad \text { for } i>j,
\end{gathered}
$$

such that $d_{i k}=\sqrt{\left\|\psi_{i}\right\|^{2}-\sum_{k=1}^{i-1} c_{i k}^{2}}, c_{i k}=\left\langle\psi_{i}, \bar{\psi}_{k}\right\rangle_{W_{2}^{5}}$, and $\left\{\psi_{i}(x)\right\}_{i=1}^{\infty}$ is the orthonormal system in the space $W_{2}^{5}[a, b]$.

Through the next theorem, the subscript $y$ by the operator $L\left(L_{y}\right)$ indicates that the operator $L$ applies to the function of $y$.

Theorem 9. If $\left\{x_{i}\right\}_{i=1}^{\infty}$ is dense on $[a, b]$, then $\left\{\psi_{i}(x)\right\}_{i=1}^{\infty}$ is a complete function system of $W_{2}^{5}[a, b]$ and $\psi_{i}(x)=$ $\left.L_{y} K_{x}(y)\right|_{y=x_{i}}$.

Proof. Clearly, $\psi_{i}(x)=L_{i}^{*} \varphi(x)=\left\langle L_{i}^{*} \varphi(y), K_{x}(y)\right\rangle_{W_{2}^{5}}=$ $\left\langle\varphi_{i}(y), L_{y} K_{x}(y)\right\rangle_{W_{2}^{1}}=\left.L_{y} K_{x}(y)\right|_{y=x_{i}} \in W_{2}^{5}[a, b]$. Now, for each fixed $u(x) \in W_{2}^{5}[a, b]$, let $\left\langle u(x), \psi_{i}(x)\right\rangle_{W_{2}^{5}}=0$, $i=1,2, \ldots$. In other word, $\left\langle u(x), \psi_{i}(x)\right\rangle_{W_{2}^{5}}=$ $\left\langle u(x), L^{*} \varphi_{i}(x)\right\rangle_{W_{2}^{5}}=\left\langle L u(x), \varphi_{i}(x)\right\rangle_{W_{2}^{1}}=L u\left(x_{i}\right)=0$. Note that $\left\{x_{i}\right\}_{i=1}^{\infty}$ is dense on $[a, b]$, and therefore $L u(x)=0$. It follows that $u(x)=0$ from the existence of $L^{-1}$. So, the proof of the theorem is complete.

Lemma 10. If $u(x) \in W_{2}^{5}[a, b]$, then there exists $M>0$ such that $\left\|u^{(i)}(x)\right\|_{C} \leq M\|u(x)\|_{W_{2}^{5}}, i=0,1,2,3,4$, where $\|u(x)\|_{C}=$ $\max _{a \leq x \leq b}|u(x)|$.

Proof. For any $x, y \in[a, b]$, we have $u^{(i)}(x)=$ $\left\langle u(y), K_{x}^{(i)}(y)\right\rangle_{W_{2}^{5}}, i=0,1,2,3,4$. By the expression of $K_{x}(y)$, it follows that $\left\|K_{x}^{(i)}(y)\right\|_{W_{2}^{5}} \leq M_{i}, i=0,1,2,3,4$. Thus, $\left|u^{(i)}(x)\right|=\left|\left\langle u(x), K_{x}^{(i)}(x)\right\rangle_{W_{2}^{5}}\right| \leq\left\|K_{x}^{(i)}(x)\right\|_{W_{2}^{5}}\|u(x)\|_{W_{2}^{5}} \leq$ $M_{i}\|u(x)\|_{W_{2}^{5}}, i=0,1,2,3,4$. Hence, $\left\|u^{(i)}(x)\right\|_{C} \leq$ $M\|u(x)\|_{W_{2}^{5}}, i=0,1,2,3,4$, where $M=\max _{i=0,1,2,3,4}\left\{M_{i}\right\}$.

The structure of the next theorem is as follows. Firstly, we will give the representation of the exact solution of (1) and (2) in the space $W_{2}^{5}[a, b]$. After that, the convergence of approximate solution $u_{n}(x)$ to the analytic solution will be proved.

Theorem 11. For each $u(x)$ in the space $W_{2}^{5}[a, b]$, the series $\sum_{i=1}^{\infty}\left\langle u(x), \bar{\psi}_{i}(x)\right\rangle \bar{\psi}_{i}(x)$ is convergent in the sense of the norm of $W_{2}^{5}[a, b]$. On the other hand, if $\left\{x_{i}\right\}_{i=1}^{\infty}$ is dense on $[a, b]$ and the solution of (1) and (2) is unique, then (i) the exact solution of (9) could be represented by

$$
u(x)=\sum_{i=1}^{\infty} \sum_{k=1}^{i} \beta_{i k} F\left(x_{k}, u\left(x_{k}\right), T u\left(x_{k}\right), S u\left(x_{k}\right)\right) \bar{\psi}_{i}(x) \text {; }
$$

(ii) the approximate solution of (9)

$$
u_{n}(x)=\sum_{i=1}^{n} \sum_{k=1}^{i} \beta_{i k} F\left(x_{k}, u\left(x_{k}\right), T u\left(x_{k}\right), S u\left(x_{k}\right)\right) \bar{\psi}_{i}(x) \text {, }
$$

and $u_{n}^{(i)}(x), i=0,1,2,3,4$ are converging uniformly to the exact solution $u(x)$ and all its derivative as $n \rightarrow$ $\infty$, respectively.

Proof. For the first part, let $u(x)$ be solution of (9) in the space $W_{2}^{5}[a, b]$. Since $u(x)$ belong to Hilbert space $W_{2}^{5}[a, b]$ and $\sum_{i=1}^{\infty}\left\langle u(x), \bar{\psi}_{i}(x)\right\rangle \bar{\psi}_{i}(x)$ is the Fourier series about normal orthogonal system $\left\{\bar{\psi}_{i}(x)\right\}_{i=1}^{\infty}$; then, the series $\sum_{i=1}^{\infty}\left\langle u(x), \bar{\psi}_{i}(x)\right\rangle \bar{\psi}_{i}(x)$ is convergent in the sense of $\|\cdot\|_{W_{2}^{5}}$. On the other hand, using (10), we have

$$
\begin{aligned}
u(x) & =\sum_{i=1}^{\infty}\left\langle u(x), \bar{\psi}_{i}(x)\right\rangle_{W_{2}^{5}} \bar{\psi}_{i}(x) \\
& =\sum_{i=1}^{\infty} \sum_{k=1}^{i} \beta_{i k}\left\langle u(x), \psi_{k}(x)\right\rangle_{W_{2}^{5}} \bar{\psi}_{i}(x) \\
& =\sum_{i=1}^{\infty} \sum_{k=1}^{i} \beta_{i k}\left\langle u(x), L^{*} \varphi_{k}(x)\right\rangle_{W_{2}^{5}} \bar{\psi}_{i}(x) \\
& =\sum_{i=1}^{\infty} \sum_{k=1}^{i} \beta_{i k}\left\langle L u(x), \varphi_{k}(x)\right\rangle_{W_{2}^{1}} \bar{\psi}_{i}(x) \\
& =\sum_{i=1}^{\infty} \sum_{k=1}^{i} \beta_{i k}\left\langle F(x, u(x), T u(x), S u(x)), \varphi_{k}(x)\right\rangle_{W_{2}^{1}} \bar{\psi}_{i}(x) \\
& =\sum_{i=1}^{\infty} \sum_{k=1}^{i} \beta_{i k} F\left(x_{k}, u\left(x_{k}\right), T u\left(x_{k}\right), S u\left(x_{k}\right)\right) \bar{\psi}_{i}(x)
\end{aligned}
$$

Therefore, the form of (12) is the exact solution of (9).

For the second part, it easy to see that by Lemma 10, for any $x \in[a, b]$,

$$
\begin{aligned}
\left|u_{n}(x)-u(x)\right| & =\left|\left\langle u_{n}(x)-u(x), K_{x}(x)\right\rangle_{W_{2}^{5}}\right| \\
& \leq\left\|K_{x}(x)\right\|_{W_{2}^{5}}\left\|u_{n}(x)-u(x)\right\|_{W_{2}^{5}} \\
& \leq M_{0}\left\|u_{n}(x)-u(x)\right\|_{W_{2}^{5}} .
\end{aligned}
$$

On the other hand,

$$
\left|u_{n}^{(i)}(x)-u^{(i)}(x)\right|=\left|\left\langle u_{n}(x)-u(x), K_{x}^{(i)}(x)\right\rangle_{W_{2}^{5}}\right|
$$




$$
\begin{aligned}
& \leq\left\|K_{x}^{(i)}(x)\right\|_{W_{2}^{5}}\left\|u_{n}(x)-u(x)\right\|_{W_{2}^{5}} \\
& \leq M_{i}\left\|u_{n}(x)-u(x)\right\|_{W_{2}^{5}}, \quad i=1,2,3,4,
\end{aligned}
$$

where $M_{i}, i=0,1,2,3,4$ are positive constants. Hence, if $\left\|u_{n}(x)-u(x)\right\|_{W_{2}^{5}} \rightarrow 0$ as $n \rightarrow \infty$, the approximate solution $u_{n}(x)$ and $u_{n}^{(i)}(x), i=0,1,2,3,4$ converge uniformly to the exact solution $u(x)$ and all its derivative, respectively. So, the proof of the theorem is complete.

Remark 12. We mention here that the approximate solution $u_{n}(x)$ in (13) can be obtained by taking finitely many terms in the series representation for $u(x)$ of (12).

\section{Procedure of Constructing Iterative Method}

In this section, an iterative method of obtaining the solution of (9) is presented in the reproducing kernel space $W_{2}^{5}[a, b]$ for both linear and nonlinear cases. Initially, we will mention the following remark about the exact and approximate solutions of (1) and (2).

Remark 13. In order to apply the RKHS technique to solve (1) and (2), we have the following two cases based on the structure of the functions $N, G_{1}$, and $G_{2}$.

Case 1. If (1) is linear, then the exact and approximate solutions can be obtained directly from (12) and (13), respectively.

Case 2. If (1) is nonlinear, then in this case the exact and approximate solutions can be obtained by using the following iterative algorithm.

Algorithm 14. According to (12), the representation of the analytical solution of (1) can be denoted by the following series:

$$
u(x)=\sum_{i=1}^{\infty} B_{i} \bar{\psi}_{i}(x)
$$

where $B_{i}=\sum_{k=1}^{i} \beta_{i k} F\left(x_{k}, u_{k-1}\left(x_{k}\right), T u_{k-1}\left(x_{k}\right), S u_{k-1}\left(x_{k}\right)\right)$. In fact, $B_{i}, i=1,2, \ldots$ in (17) are unknown; we will approximate $B_{i}$ using known $A_{i}$. For numerical computations, we define initial function $u_{0}\left(x_{1}\right)=0$, put $u_{0}\left(x_{1}\right)=u\left(x_{1}\right)$, and define the $n$-term approximation to $u(x)$ by

$$
u_{n}(x)=\sum_{i=1}^{n} A_{i} \bar{\psi}_{i}(x) \text {, }
$$

where the coefficients $A_{i}$ of $\bar{\psi}_{i}(x), i=1,2, \ldots, n$ are given as follows:

$$
\begin{gathered}
A_{1}=\beta_{11} F\left(x_{1}, u_{0}\left(x_{1}\right), T u_{0}\left(x_{1}\right), S u_{0}\left(x_{1}\right)\right), \\
u_{1}(x)=A_{1} \bar{\psi}_{1}(x), \\
A_{2}=\sum_{k=1}^{2} \beta_{2 k} F\left(x_{k}, u_{k-1}\left(x_{k}\right), T u_{k-1}\left(x_{k}\right), S u_{k-1}\left(x_{k}\right)\right),
\end{gathered}
$$

$$
u_{2}(x)=\sum_{i=1}^{2} A_{i} \bar{\psi}_{i}(x)
$$

$\cdots$

$$
\begin{gathered}
u_{n-1}(x)=\sum_{i=1}^{n-1} A_{i} \bar{\psi}_{i}(x) \\
A_{n}=\sum_{k=1}^{n} \beta_{n k} F\left(x_{k}, u_{k-1}\left(x_{k}\right), T u_{k-1}\left(x_{k}\right), S u_{k-1}\left(x_{k}\right)\right) .
\end{gathered}
$$

Here, note that in the iterative process of (18), we can guarantee that the approximation $u_{n}(x)$ satisfies the boundary conditions (2). Now, the approximate solution $u_{n}^{N}(x)$ can be obtained by taking finitely many terms in the series representation of $u_{n}(x)$ and

$$
\begin{aligned}
& u_{n}^{N}(x) \\
& =\sum_{i=1}^{N} \sum_{k=1}^{i} \beta_{i k} F\left(x_{k}, u_{n-1}\left(x_{k}\right), T u_{n-1}\left(x_{k}\right), S u_{n-1}\left(x_{k}\right)\right) \bar{\psi}_{i}(x) .
\end{aligned}
$$

Next, we will prove that $u_{n}(x)$ in the iterative formula (18) converges to the exact solution $u(x)$ of (1); in fact, this result is fundamental in the RKHS theory and its applications. The next two lemmas are collected for future use in order to prove the recent theorem.

Lemma 15. If $u_{n}(x) \rightarrow u(x)$ in the sense of the norm of $W_{2}^{5}[a, b], x_{n} \rightarrow y$ as $n \rightarrow \infty$, and $F(x, v, w, z)$ is continuous in $[a, b]$ with respect to $x, v, w, z$ for $x \in[a, b]$ and $v, w, z \in$ $(-\infty, \infty)$, then $F\left(x_{n}, u_{n-1}\left(x_{n}\right), T u_{n-1}\left(x_{n}\right), S u_{n-1}\left(x_{n}\right)\right) \rightarrow$ $F(y, u(y), T u(y), \operatorname{Su}(y))$ as $n \rightarrow \infty$.

Proof. Firstly, we will prove that $u_{n-1}\left(x_{n}\right) \rightarrow u(y)$ in the sense of $\|\cdot\|_{W_{2}^{5}}$, since

$$
\begin{aligned}
\mid u_{n-1} & \left(x_{n}\right)-u(y) \mid \\
& =\left|u_{n-1}\left(x_{n}\right)-u_{n-1}(y)+u_{n-1}(y)-u(y)\right| \\
& \leq\left|u_{n-1}\left(x_{n}\right)-u_{n-1}(y)\right|+\left|u_{n-1}(y)-u(y)\right| .
\end{aligned}
$$

By reproducing property of $K_{x}(y)$, we have $u_{n-1}\left(x_{n}\right)=$ $\left\langle u_{n-1}(x), K_{x_{n}}(x)\right\rangle$ and $u_{n-1}(y)=\left\langle u_{n-1}(x), K_{y}(x)\right\rangle$. Thus, $\left|u_{n-1}\left(x_{n}\right)-u_{n-1}(y)\right|=\left|\left\langle u_{n-1}(x), K_{x_{n}}(x)-K_{y}(x)\right\rangle_{W_{2}^{5}}\right| \leq$ $\left\|u_{n-1}(x)\right\|_{W_{2}^{5}}\left\|K_{x_{n}}(x)-K_{y}(x)\right\|_{W_{2}^{5}}$. From the symmetry of $K_{x}(y)$, it follows that $\left\|K_{x_{n}}(x)-K_{y}(x)\right\|_{W_{2}^{5}} \rightarrow 0$ as $n \rightarrow \infty$. Hence, $\left|u_{n-1}\left(x_{n}\right)-u_{n-1}(y)\right| \rightarrow 0$ as soon as $x_{n} \rightarrow y$. On the other hand, by Theorem 11 part (ii), for any $y \in[a, b]$, it holds that $\left|u_{n-1}(y)-u(y)\right| \rightarrow 0$ as $n \rightarrow \infty$. Therefore, $u_{n-1}\left(x_{n}\right) \rightarrow u(y)$ in the sense of $\|\cdot\|_{W_{2}^{5}}$ as $x_{n} \rightarrow y$ and $n \rightarrow \infty$.

Thus, by means of the continuation of $G_{1}, G_{2}$, and $N$, it is obtained that $G_{1}\left(u_{n-1}\left(x_{n}\right)\right) \rightarrow G_{1}(u(y)), G_{2}\left(u_{n-1}\left(x_{n}\right)\right) \rightarrow$ $G_{2}(u(y))$, and $N\left(x_{n}, u_{n-1}\left(x_{n}\right)\right) \rightarrow N(y, u(y))$ as $n \rightarrow \infty$. This shows that $T u_{n-1}\left(x_{n}\right) \rightarrow T u(y)$ and $S u_{n-1}\left(x_{n}\right) \rightarrow$ $\operatorname{Su}(y)$ as $n \rightarrow \infty$. Hence, the continuity of $F$ gives the result. 
Lemma 16. For $j \leq n$, one has

$$
\begin{aligned}
\operatorname{Lu}\left(x_{j}\right) & =\operatorname{Lu}\left(x_{j}\right) \\
& =F\left(x_{j}, u_{j-1}\left(x_{j}\right), T u_{j-1}\left(x_{j}\right), S u_{j-1}\left(x_{j}\right)\right) .
\end{aligned}
$$

Proof. The proof of

$$
L u_{n}\left(x_{j}\right)=F\left(x_{j}, u_{j-1}\left(x_{j}\right), T u_{j-1}\left(x_{j}\right), S u_{j-1}\left(x_{j}\right)\right)
$$

will be obtained by induction as follows:

If $j \leq n$, then

$$
\begin{aligned}
L u_{n}\left(x_{j}\right) & =\sum_{i=1}^{n} A_{i} L \bar{\psi}_{i}\left(x_{j}\right) \\
& =\sum_{i=1}^{n} A_{i}\left\langle L \bar{\psi}_{i}(x), \varphi_{j}(x)\right\rangle_{W_{2}^{1}} \\
& =\sum_{i=1}^{n} A_{i}\left\langle\bar{\psi}_{i}(x), L_{j}^{*} \varphi(x)\right\rangle_{W_{2}^{5}} \\
& =\sum_{i=1}^{n} A_{i}\left\langle\bar{\psi}_{i}(x), \psi_{j}(x)\right\rangle_{W_{2}^{5}} .
\end{aligned}
$$

Using the orthogonality of $\left\{\bar{\psi}_{i}(x)\right\}_{i=1}^{\infty}$ yields that

$$
\begin{aligned}
\sum_{l=1}^{j} \beta_{j l} L u_{n}\left(x_{l}\right) & =\sum_{i=1}^{n} A_{i}\left\langle\bar{\psi}_{i}(x), \sum_{l=1}^{j} \beta_{j l} \psi_{l}(x)\right\rangle_{W_{2}^{5}} \\
& =\sum_{i=1}^{n} A_{i}\left\langle\bar{\psi}_{i}(x), \bar{\psi}_{j}(x)\right\rangle_{W_{2}^{5}}=A_{j} \\
& =\sum_{l=1}^{j} \beta_{j l} F\left(x_{l}, u_{l-1}\left(x_{l}\right), T u_{l-1}\left(x_{l}\right), S u_{l-1}\left(x_{l}\right)\right) .
\end{aligned}
$$

Now, if $j=1$, then

$$
L u_{n}\left(x_{1}\right)=F\left(x_{1}, u_{0}\left(x_{1}\right), T u_{0}\left(x_{1}\right), S u_{0}\left(x_{1}\right)\right) .
$$

Again, if $j=2$, then

$$
\begin{aligned}
& \beta_{21} L u_{n}\left(x_{1}\right)+\beta_{22} L u_{n}\left(x_{2}\right) \\
& =\beta_{21} F\left(x_{1}, u_{0}\left(x_{1}\right), T u_{0}\left(x_{1}\right), S u_{0}\left(x_{1}\right)\right) \\
& +\beta_{22} F\left(x_{2}, u_{1}\left(x_{2}\right), T u_{1}\left(x_{2}\right), S u_{1}\left(x_{2}\right)\right) .
\end{aligned}
$$

Thus, $L u_{n}\left(x_{2}\right)=F\left(x_{2}, u_{1}\left(x_{2}\right), T u_{1}\left(x_{2}\right), S u_{1}\left(x_{2}\right)\right)$. It is easy to see by using mathematical induction concept that $L u_{n}\left(x_{j}\right)=F\left(x_{j}, u_{j-1}\left(x_{j}\right), T u_{j-1}\left(x_{j}\right), S u_{j-1}\left(x_{j}\right)\right)$.

On the other hand, from Theorem $11, u_{n}(x)$ converge uniformly to $u(x)$. It follows that on taking limits in (18) $u(x)=$ $\sum_{i=1}^{\infty} B_{i} \bar{\psi}_{i}(x)$. Therefore, $u_{n}(x)=P_{n} u(x)$, where $P_{n}$ is an orthogonal projector from $W_{2}^{5}[a, b]$ to $\operatorname{Span}\left\{\psi_{1}, \psi_{2}, \ldots, \psi_{n}\right\}$. Thus, $L u_{n}\left(x_{j}\right)=\left\langle\operatorname{Lu}_{n}(x), \varphi_{j}(x)\right\rangle_{W_{2}^{1}}=\left\langle u_{n}(x), L_{j}^{*} \varphi(x)\right\rangle_{W_{2}^{5}}=$ $\left\langle P_{n} u(x), \psi_{j}(x)\right\rangle_{W_{2}^{5}}=\left\langle u(x), P_{n} \psi_{j}(x)\right\rangle_{W_{2}^{5}}=\left\langle u(x), \psi_{j}(x)\right\rangle_{W_{2}^{5}}=$ $\left\langle\operatorname{Lu}(x), \varphi_{j}(x)\right\rangle_{W_{2}^{1}}=\operatorname{Lu}\left(x_{j}\right)$.
Theorem 17. If $\left\{x_{i}\right\}_{i=1}^{\infty}$ is dense on $[a, b]$ and $\left\|u_{n}\right\|_{W_{2}^{5}}$ is bound$e d$, then the $n$-term approximate solution $u_{n}(x)$ in the iterative formula (18) converges to the exact solution $u(x)$ of (9) in the space $W_{2}^{5}[a, b]$ and $u(x)=\sum_{i=1}^{\infty} A_{i} \bar{\psi}_{i}(x)$, where $A_{i}$ are given by (19).

Proof. The proof consists of the following three steps. Firstly, we will prove that the sequence $\left\{u_{n}\right\}_{n=1}^{\infty}$ in (18) is monotone increasing in the sense of $\|\cdot\|_{W_{2}^{5}}$. By Theorem 9, $\left\{\bar{\psi}_{i}\right\}_{i=1}^{\infty}$ is the complete orthonormal system in $W_{2}^{5}[a, b]$. Hence, we have $\left\|u_{n}\right\|_{W_{2}^{5}}^{2}=\left\langle u_{n}(x), u_{n}(x)\right\rangle_{W_{2}^{5}}=\left\langle\sum_{i=1}^{n} A_{i} \bar{\psi}_{i}(x)\right.$, $\left.\sum_{i=1}^{n} A_{i} \bar{\psi}_{i}(x)\right\rangle_{W_{2}^{5}}=\sum_{i=1}^{n}\left(A_{i}\right)^{2}$. Therefore, $\left\|u_{n}\right\|_{W_{2}^{5}}$ is monotone increasing.

Secondly, we will prove the convergence of $u_{n}(x)$. From (18), we have $u_{n+1}(x)=u_{n}(x)+A_{n+1} \bar{\psi}_{n+1}(x)$. From the orthogonality of $\left\{\bar{\psi}_{i}(x)\right\}_{i=1}^{\infty}$, it follows that $\left\|u_{n+1}\right\|_{W_{2}^{5}}^{2}=$ $\left\|u_{n}\right\|_{W_{2}^{5}}^{2}+\left(A_{n+1}\right)^{2}=\left\|u_{n-1}\right\|_{W_{2}^{5}}^{2}+\left(A_{n}\right)^{2}+\left(A_{n+1}\right)^{2}=$ $\cdots=\left\|u_{0}\right\|_{W_{2}^{5}}^{2}+\sum_{i=1}^{n+1}\left(A_{i}\right)^{2}$. Since, the sequence $\left\|u_{n}\right\|_{W_{2}^{5}}$ is monotone increasing. Due to the condition that $\left\|u_{n}\right\|_{W_{2}^{5}}$ is bounded, $\left\|u_{n}\right\|_{W_{2}^{5}}$ is convergent as $n \rightarrow \infty$. Then, there exists a constant $c$ such that $\sum_{i=1}^{\infty}\left(A_{i}\right)^{2}=c$. It implies that $A_{i}=\sum_{k=1}^{i} \beta_{i k} F\left(x_{k}, u_{k-1}\left(x_{k}\right), T u_{k-1}\left(x_{k}\right), S u_{k-1}\left(x_{k}\right)\right) \in l^{2}, i=$ $1,2, \ldots$. On the other hand, since $\left(u_{m}-u_{m-1}\right) \perp\left(u_{m-1}-\right.$ $\left.u_{m-2}\right) \perp \cdots \perp\left(u_{n+1}-u_{n}\right)$, it follows for $m>n$ that

$$
\begin{aligned}
& \left\|u_{m}(x)-u_{n}(x)\right\|_{W_{2}^{5}}^{2} \\
& =\left\|u_{m}(x)-u_{m-1}(x)+u_{m-1}(x)-\cdots+u_{n+1}(x)-u_{n}(x)\right\|_{W_{2}^{5}}^{2} \\
& =\left\|u_{m}(x)-u_{m-1}(x)\right\|_{W_{2}^{5}}^{2}+\cdots+\left\|u_{n+1}(x)-u_{n}(x)\right\|_{W_{2}^{5}}^{2} .
\end{aligned}
$$

Furthermore, $\left\|u_{m}(x)-u_{m-1}(x)\right\|_{W_{2}^{5}}^{2}=\left(A_{m}\right)^{2}$. Consequently, as $n, m \rightarrow \infty$, we have $\left\|u_{m}(x)-u_{n}(x)\right\|_{W_{2}^{5}}^{2}=\sum_{i=n+1}^{m}\left(A_{i}\right)^{2} \rightarrow$ 0 . Considering the completeness of $W_{2}^{5}[a, b]$, there exists a $u(x) \in W_{2}^{5}[a, b]$ such that $u_{n}(x) \rightarrow u(x)$ as $n \rightarrow \infty$ in the sense of $\|\cdot\|_{W_{2}^{5}}$.

Thirdly, we will prove that $u(x)$ is the solution of (9). Since $\left\{x_{i}\right\}_{i=1}^{\infty}$ is dense on $[a, b]$, for any $x \in[a, b]$, there exists subsequence $\left\{x_{n_{j}}\right\}_{j=1}^{\infty}$, such that $x_{n_{j}} \rightarrow x$ as $j \rightarrow \infty$. From Lemma 16, it is clear that $\operatorname{Lu}\left(x_{n_{j}}\right)=F\left(x_{n_{j}}, u_{n_{j}-1}\left(x_{k}\right)\right.$, $\left.T u_{n_{j}-1}\left(x_{k}\right), S u_{n_{j}-1}\left(x_{k}\right)\right)$. Hence, let $j \rightarrow \infty$, by Lemma 15 and the continuity of $F$, we have $L u(x)=F(x, u(x), T u(x), S u(x))$. That is, $u(x)$ satisfies (1). Also, since $\bar{\psi}_{i}(x) \in W_{2}^{5}[a, b]$, clearly, $u(x)$ satisfies the boundary conditions (2). In other words, $u(x)$ is the solution of (1) and (2), where $u(x)=\sum_{i=1}^{\infty} A_{i} \bar{\psi}_{i}(x)$ and $A_{i}$ are given by (19). The proof is complete.

It obvious that if we let $u(x)$ denote the exact solution of (9), $u_{n}(x)$ the approximate solution obtained by the RKHS method as given by (18), and $r_{n}(x)$ the difference between $u(x)$ and $u_{n}(x)$, where $x \in[a, b]$, then $\left\|r_{n}(x)\right\|_{W_{2}^{5}}^{2}=$ $\left\|u(x)-u_{n}(x)\right\|_{W_{2}^{5}}^{2}=\left\|\sum_{i=n+1}^{\infty} A_{i} \bar{\psi}_{i}(x)\right\|_{W_{2}^{5}}^{2}=\sum_{i=n+1}^{\infty}\left(A_{i}\right)^{2}$ and 
TABLE 1: Numerical results for Example 19.

\begin{tabular}{lcccc}
\hline$x$ & Exact solution & Approximate solution & Absolute error & Relative error \\
\hline 0.16 & -1.68 & -1.679999999248148 & $7.51852 \times 10^{-10}$ & $4.47531 \times 10^{-10}$ \\
0.32 & -1.36 & -1.359999998030627 & $1.96937 \times 10^{-9}$ & $1.44807 \times 10^{-9}$ \\
0.48 & -1.04 & -1.039999997409132 & $2.59087 \times 10^{-9}$ & $2.49122 \times 10^{-9}$ \\
0.64 & -0.72 & -0.719999997792201 & $2.20780 \times 10^{-9}$ & $3.06639 \times 10^{-9}$ \\
0.80 & -0.40 & -0.399999998934791 & $1.06521 \times 10^{-9}$ & $2.66302 \times 10^{-9}$ \\
0.96 & -0.08 & -0.079999999937888 & $6.21123 \times 10^{-11}$ & $7.76404 \times 10^{-10}$ \\
\hline
\end{tabular}

TABLE 2: Numerical results for Example 20.

\begin{tabular}{lcccc}
\hline$x$ & Exact solution & Approximate solution & Absolute error & Relative error \\
\hline 0.16 & 0.0256 & 0.025600007512175 & $7.51217 \times 10^{-9}$ & $2.93444 \times 10^{-7}$ \\
0.32 & 0.1024 & 0.102400019690182 & $1.96902 \times 10^{-8}$ & $1.92287 \times 10^{-7}$ \\
0.48 & 0.2304 & 0.230400025906381 & $2.59064 \times 10^{-8}$ & $1.12441 \times 10^{-7}$ \\
0.64 & 0.4096 & 0.409600022074531 & $2.20745 \times 10^{-8}$ & $5.38929 \times 10^{-8}$ \\
0.80 & 0.6400 & 0.640000010646264 & $1.06463 \times 10^{-8}$ & $1.66348 \times 10^{-8}$ \\
0.96 & 0.9216 & 0.921600000613291 & $6.13291 \times 10^{-10}$ & $6.65463 \times 10^{-10}$ \\
\hline
\end{tabular}

$\left\|r_{n-1}(x)\right\|_{W_{2}^{5}}^{2}=\sum_{i=n}^{\infty}\left(A_{i}\right)^{2}$ or $\left\|r_{n}(x)\right\|_{W_{2}^{5}} \leq\left\|r_{n-1}(x)\right\|_{W_{2}^{5}}$. In fact, this is just the proof of the following theorem.

Theorem 18. The difference function $r_{n}(x)$ is monotone decreasing in the sense of the norm of $W_{2}^{5}[a, b]$.

\section{Numerical Outcomes}

In this section, we propose few numerical simulations implemented by Mathematica 7.0 software package for solving some specific examples of (1) and (2). However, we apply the techniques described in the previous sections to some linear and nonlinear test examples in order to demonstrate the efficiency, accuracy, and applicability of the proposed method. Results obtained by the method are compared with the analytical solution of each example by computing the exact and relative errors and are found to be in good agreement with each other.

Example 19. Consider the following linear Fredholm-Volterra IDE:

$$
\begin{aligned}
u^{(4)}(x) & +u(x)-\int_{0}^{1}(x t) u(t) d t-\int_{0}^{x} x(t+1) u(t) d t \\
= & f(x), \quad 0 \leq x, t \leq 1,
\end{aligned}
$$

subject to the boundary conditions

$$
\begin{array}{ll}
u(0)=-2, & u(1)=0, \\
u^{\prime}(0)=2, & u^{\prime}(1)=2,
\end{array}
$$

where $f(x)=-(2 / 3) x^{4}+2 x^{2}+(7 / 3) x-2$. The exact solution is $u(x)=2 x-2$.

Using RKHS method, taking $x_{i}=(i-1) /(n-1), i=$ $1,2, \ldots, n$ with the reproducing kernel function $K_{x}(y)$ on
$[0,1]$, the approximate solution $u_{n}(x)$ is calculated by (13). The numerical results at some selected grid points for $n=26$ are given in Table 1.

Example 20. Consider the following nonlinear FredholmVolterra IDE:

$$
\begin{aligned}
u^{(4)}(x) & +u(x)-\frac{1}{4} \int_{0}^{1}(t) u^{3}(t) d t+\frac{1}{2} \int_{0}^{x}(x) u^{2}(t) d t \\
= & f(x), \quad 0 \leq x, t \leq 1,
\end{aligned}
$$

subject to the boundary conditions

$$
\begin{array}{cc}
u(0)=0, & u(1)=1, \\
u^{\prime}(0)=0, & u^{\prime}(1)=2,
\end{array}
$$

where $f(x)=(1 / 10) x^{6}+x^{2}-(1 / 32)$. The exact solution is $u(x)=x^{2}$.

Using RKHS method, taking $x_{i}=(i-1) /(N-1), i=$ $1,2, \ldots, N$ with the reproducing kernel function $K_{x}(y)$ on $[0,1]$, the approximate solution $u_{n}^{N}(x)$ is calculated by $(20)$. The numerical results at some selected grid points for $N=11$ and $n=3$ are given in Table 2 .

Example 21. Consider the following nonlinear FredholmVolterra IDE:

$$
\begin{aligned}
u^{(4)}(x) & +u^{2}(x)-\int_{0}^{1}(t+1) \sinh (u(t)) d t-\int_{0}^{x}(x) e^{u(t)} d t \\
= & f(x), \quad 0 \leq x, t \leq 1,
\end{aligned}
$$

subject to the boundary conditions

$$
\begin{gathered}
u(0)=0, \quad u(1)=\ln (2), \\
u^{\prime}(0)=1, \quad u^{\prime}(1)=\frac{1}{2},
\end{gathered}
$$


TABLE 3: Numerical results for Example 21.

\begin{tabular}{lcccc}
\hline$x$ & Exact solution & Approximate solution & Absolute error & Relative error \\
\hline 0.16 & 0.148420005118273 & 0.148419864687877 & $1.40430 \times 10^{-7}$ & $9.46169 \times 10^{-7}$ \\
0.32 & 0.277631736598280 & 0.277631416799765 & $3.19799 \times 10^{-7}$ & $1.15188 \times 10^{-6}$ \\
0.48 & 0.392042087776024 & 0.392041716651615 & $3.71124 \times 10^{-7}$ & $9.46644 \times 10^{-7}$ \\
0.64 & 0.494696241836107 & 0.494695959934014 & $2.81902 \times 10^{-7}$ & $5.69849 \times 10^{-7}$ \\
0.80 & 0.587786664902119 & 0.587786542757544 & $1.22145 \times 10^{-7}$ & $2.07804 \times 10^{-7}$ \\
0.96 & 0.672944473242426 & 0.672944466882463 & $6.35996 \times 10^{-9}$ & $9.45095 \times 10^{-9}$ \\
\hline
\end{tabular}

TABLE 4: Numerical results for Example 22.

\begin{tabular}{lcccc}
\hline$x$ & Exact solution & Approximate solution & Absolute error & Relative error \\
\hline 0.16 & 0.84 & 0.840000000735357 & $7.35357 \times 10^{-10}$ & $8.75425 \times 10^{-10}$ \\
0.32 & 0.68 & 0.680000002094899 & $2.09490 \times 10^{-9}$ & $3.08073 \times 10^{-9}$ \\
0.48 & 0.52 & 0.520000002788682 & $2.78868 \times 10^{-9}$ & $5.36285 \times 10^{-9}$ \\
0.64 & 0.36 & 0.360000002335676 & $2.33568 \times 10^{-9}$ & $6.48799 \times 10^{-9}$ \\
0.80 & 0.20 & 0.200000001005203 & $1.00520 \times 10^{-9}$ & $5.02601 \times 10^{-9}$ \\
0.96 & 0.04 & 0.039999999826935 & $1.73065 \times 10^{-10}$ & $4.32663 \times 10^{-9}$ \\
\hline
\end{tabular}

where $f(x)=(\ln (x+1))^{2}-\left(6 /(x+1)^{4}\right)-(1 / 2) x^{2}(x+2)-$ $(2 / 3)$. The exact solution is $u(x)=\ln (x+1)$.

Using RKHS method, taking $x_{i}=(i-1) /(N-1), i=$ $1,2, \ldots, N$ with the reproducing kernel function $K_{x}(y)$ on $[0,1]$, the approximate solution $u_{n}^{N}(x)$ is calculated by $(20)$. The numerical results at some selected grid points for $N=26$ and $n=5$ are given in Table 3 .

Example 22. Consider the following nonlinear FredholmVolterra IDE:

$$
\begin{gathered}
u^{(4)}(x)+\cos (u(x))-\int_{0}^{1}(t+x) u(t) d t-\int_{0}^{x} e^{t-x} e^{u(t)} d t \\
=f(x), \quad 0 \leq x, \quad t \leq 1,
\end{gathered}
$$

subject to the boundary conditions

$$
\begin{array}{cc}
u(0)=1, & u(1)=0, \\
u^{\prime}(0)=-1, & u^{\prime}(1)=-1,
\end{array}
$$

where $f(x)=\cos (1-x)-x e^{1-x}-(1 / 2) x-(1 / 6)$. The exact solution is $u(x)=1-x$.

Using RKHS method, taking $x_{i}=(i-1) /(N-1), i=$ $1,2, \ldots, N$ with the reproducing kernel function $K_{x}(y)$ on $[0,1]$, the approximate solution $u_{n}^{N}(x)$ is calculated by $(20)$. The numerical results at some selected grid points for $N=26$ and $n=5$ are given in Table 4 .

Example 23. Consider the following nonlinear FredholmVolterra IDE:

$$
\begin{aligned}
u^{(4)}(x) & +u(x)-\int_{0}^{1} \cos ^{-1}(u(t)) d t \\
- & \int_{0}^{x}(x+t)\left(1-u^{2}(t)\right) d t=f(x), \quad 0 \leq x, t \leq 1,
\end{aligned}
$$

subject to the boundary conditions

$$
\begin{aligned}
u(0)=1, & u(1)=\cos (1), \\
u^{\prime}(0)=0, & u^{\prime}(1)=-\sin (1),
\end{aligned}
$$

where $f(x)=2 \cos (x)+(1 / 2) x \sin (2 x)-(1 / 4) \sin ^{2}(x)-$ $(3 / 4) x^{2}-(1 / 2)$. The exact solution is $u(x)=\cos (x)$.

Using RKHS method, taking $x_{i}=(i-1) /(N-1), i=$ $1,2, \ldots, N$ with the reproducing kernel function $K_{x}(y)$ on $[0,1]$, the approximate solution $u_{n}^{N}(x)$ is calculated by $(20)$. The numerical results at some selected grid points for $N=26$ and $n=3$ are given in Table 5 .

As we mention earlier, it is possible to pick any point in $[a, b]$, and the approximate solutions and all its derivative up to order four will be applicable as well. Next, new numerical results for Example 23 which include the absolute error at some selected nodes in $[0,1]$ for $u^{(m)}(x), m=0,1,2,3,4$, where $x_{i}=(i-1) /(N-1), i=1,2, \ldots, N$ in which $N=26$ and $n=5$ are given in Table 6 .

\section{Conclusions}

The main concern of this work has been to propose an efficient algorithm for the solutions of two-point BVPs for fourth-order Fredholm-Volterra IDEs (1) and (2). The main goal has been achieved by introducing the RKHS method to solve this class of IDEs. We can conclude that the RKHS method is powerful and efficient technique in finding approximate solution for linear and nonlinear problems. In the proposed algorithm, the solution $u(x)$ and the approximate solution $u_{n}(x)$ are represented in the form of series in $W_{2}^{5}[a, b]$. Moreover, the approximate solution and all its derivatives converge uniformly to the exact solution and all its derivatives up to order four, respectively. There is an important point to make here; the results obtained by the 
TABLE 5: Numerical results for Example 23.

\begin{tabular}{lcccc}
\hline$x$ & Exact solution & Approximate solution & Absolute error & Relative error \\
\hline 0.16 & 0.987227283375627 & 0.987227193344231 & $9.00314 \times 10^{-8}$ & $9.11962 \times 10^{-8}$ \\
0.32 & 0.949235418082441 & 0.949235185492173 & $2.32590 \times 10^{-7}$ & $2.45029 \times 10^{-7}$ \\
0.48 & 0.886994922779284 & 0.886994621805469 & $3.00974 \times 10^{-7}$ & $3.39319 \times 10^{-7}$ \\
0.64 & 0.802095757884293 & 0.802095506191996 & $2.51692 \times 10^{-7}$ & $3.13793 \times 10^{-7}$ \\
0.80 & 0.696706709347165 & 0.696706590465593 & $1.18882 \times 10^{-7}$ & $1.70634 \times 10^{-7}$ \\
0.96 & 0.573519986072450 & 0.573519979379673 & $6.69278 \times 10^{-9}$ & $1.16697 \times 10^{-8}$ \\
\hline
\end{tabular}

TABLE 6: Absolute error of $u^{(m)}(x)$ for Example 23.

\begin{tabular}{lcccc}
\hline$m$ & $x=0.16$ & $x=0.48$ & $x=0.64$ & $x=0.96$ \\
\hline 0 & $9.00314 \times 10^{-8}$ & $3.00974 \times 10^{-7}$ & $2.51692 \times 10^{-7}$ & $6.69278 \times 10^{-9}$ \\
1 & $2.31757 \times 10^{-7}$ & $9.03440 \times 10^{-7}$ & $6.42840 \times 10^{-7}$ & $3.21697 \times 10^{-8}$ \\
2 & $1.77287 \times 10^{-6}$ & $4.84667 \times 10^{-6}$ & $3.48865 \times 10^{-6}$ & $7.08367 \times 10^{-7}$ \\
3 & $5.13644 \times 10^{-6}$ & $2.04982 \times 10^{-5}$ & $1.74082 \times 10^{-5}$ & $4.70439 \times 10^{-6}$ \\
4 & $1.00313 \times 10^{-5}$ & $3.00974 \times 10^{-5}$ & $2.51692 \times 10^{-5}$ & $6.69283 \times 10^{-6}$ \\
\hline
\end{tabular}

RKHS method are very effective and convenient in linear and nonlinear cases with less computational, iteration steps, work, and time. This confirms our belief that the efficiency of our technique gives it much wider applicability in the future for general classes of linear and nonlinear problems.

\section{References}

[1] F. Bloom, "Asymptotic bounds for solutions to a system of damped integro-differential equations of electromagnetic theory," Journal of Mathematical Analysis and Applications, vol. 73, no. 2, pp. 524-542, 1980.

[2] K. Holmåker, "Global asymptotic stability for a stationary solution of a system of integro-differential equations describing the formation of liver zones," SIAM Journal on Mathematical Analysis, vol. 24, no. 1, pp. 116-128, 1993.

[3] L. K. Forbes, S. Crozier, and D. M. Doddrell, "Calculating current densities and fields produced by shielded magnetic resonance imaging probes," SIAM Journal on Applied Mathematics, vol. 57, no. 2, pp. 401-425, 1997.

[4] R. P. Kanwal, Linear Integral differential Equations Theory and Technique, Academic Press, New York, NY, USA, 1971.

[5] A.-M. Wazwaz, "A reliable algorithm for solving boundary value problems for higher-order integro-differentiable equations," Applied Mathematics and Computation, vol. 118, no. 2-3, pp. 327342, 2001.

[6] A. Arikoglu and I. Ozkol, "Solution of boundary value problems for integro-differential equations by using differential transform method," Applied Mathematics and Computation, vol. 168, no. 2, pp. 1145-1158, 2005.

[7] A. Yildırım, "Solution of BVPs for fourth-order integro-differential equations by using homotopy perturbation method," Computers \& Mathematics with Applications, vol. 56, no. 12, pp. 3175-3180, 2008.

[8] M. Saeidy, M. Matinfar, and J. Vahidi, "Analytical solution of BVPs for fourth-order integro-differential equations by using homotopy analysis method," International Journal of Nonlinear Science, vol. 9, no. 4, pp. 414-421, 2010.
[9] O. Abu Arqub, "Series solution of fuzzy differential equations under strongly generalized di erentiability," Journal of Advanced Research in Applied Mathematics, vol. 5, pp. 31-52, 2013.

[10] O. Abu Arqub, A. El-Ajou, S. Momani, and N. Shawagfeh, "Analytical solutions of fuzzy initial value problems by HAM," Applied Mathematics and Information Sciences. In press.

[11] O. Abu Arqub and A. El-Ajou, "Solution of the fractional epidemic model by homotopy analysis method," Journal of King Saud University (Science), vol. 25, pp. 73-81, 2013.

[12] S. Momani and R. Qaralleh, "An efficient method for solving systems of fractional integro-differential equations," Journal of Mathematical Analysis and Applications, vol. 52, no. 3-4, pp. 459-470, 2006.

[13] A. El-Ajou, O. Abu Arqub, and S. Momani, "Homotopy analysis method for second-order boundary value problems of integrodifferential equations," Discrete Dynamics in Nature and Society, vol. 2012, Article ID 365792, 18 pages, 2012.

[14] O. Abu Arqub, Z. Abo-Hammour, and S. Momani, "Application of continuous genetic algorithm for nonlinear system of second-order boundary value problems," Applied Mathematics and Information Sciences. In press.

[15] O. Abu Arqub, Z. Abo-Hammour, S. Momani, and N. Shawagfeh, "Solving singular two-point boundary value problems using continuous genetic algorithm," Abstract and Applied Analysis, vol. 2012, Article ID 205391, 25 pages, 2012.

[16] A. Berlinet and C. Thomas-Agnan, Reproducing Kernel Hilbert Spaces in Probability and Statistics, Kluwer Academic Publishers, Boston, Mass, USA, 2004.

[17] M. Cui and Y. Lin, Nonlinear Numerical Analysis in the Reproducing Kernel Space, Nova Science, New York, NY, USA, 2009.

[18] D. Alpay, Ed., Reproducing Kernel Spaces and Applications, vol. 143 of Operator Theory: Advances and Applications, Birkhäuser, Basel, Switzerland, 2003.

[19] C.-1. Li and M.-g. Cui, "The exact solution for solving a class nonlinear operator equations in the reproducing kernel space," Applied Mathematics and Computation, vol. 143, no. 2-3, pp. 393-399, 2003.

[20] F. Geng and M. Cui, "Solving a nonlinear system of second order boundary value problems," Journal of Mathematical Analysis and Applications, vol. 327, no. 2, pp. 1167-1181, 2007. 
[21] F. Geng, "A new reproducing kernel Hilbert space method for solving nonlinear fourth-order boundary value problems," Applied Mathematics and Computation, vol. 213, no. 1, pp. 163$169,2009$.

[22] Y. Li, F. Geng, and M. Cui, "The analytical solution of a system of nonlinear differential equations," International Journal of Mathematical Analysis, vol. 1, no. 9-12, pp. 451-462, 2007.

[23] F. Geng, "Solving singular second order three-point boundary value problems using reproducing kernel Hilbert space method," Applied Mathematics and Computation, vol. 215, no. 6, pp. 2095-2102, 2009.

[24] Y. Z. Lin, M. G. Cui, and L. H. Yang, "Representation of the exact solution for a kind of nonlinear partial differential equation," Applied Mathematics Letters, vol. 19, no. 8, pp. 808-813, 2006.

[25] M. Cui and H. Du, "Representation of exact solution for the nonlinear Volterra-Fredholm integral equations," Applied Mathematics and Computation, vol. 182, no. 2, pp. 1795-1802, 2006.

[26] M. Al-Smadi, O. Abu Arqub, and N. Shawagfeh, "Approximate solution of BVPs for 4th-order IDEs by using RKHS method," Applied Mathematical Sciences, vol. 6, no. 49-52, pp. 2453-2464, 2012.

[27] O. Abu Arqub, M. Al-Smadi, and S. Momani, "Application of reproducing kernel method for solving nonlinear FredholmVolterra integrodifferential equations," Abstract and Applied Analysis, Article ID 839836, 16 pages, 2012. 


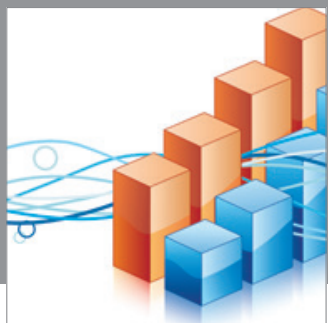

Advances in

Operations Research

mansans

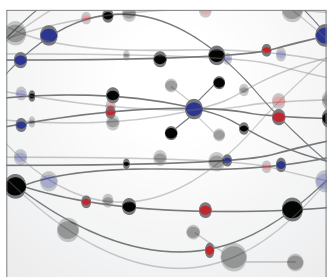

The Scientific World Journal
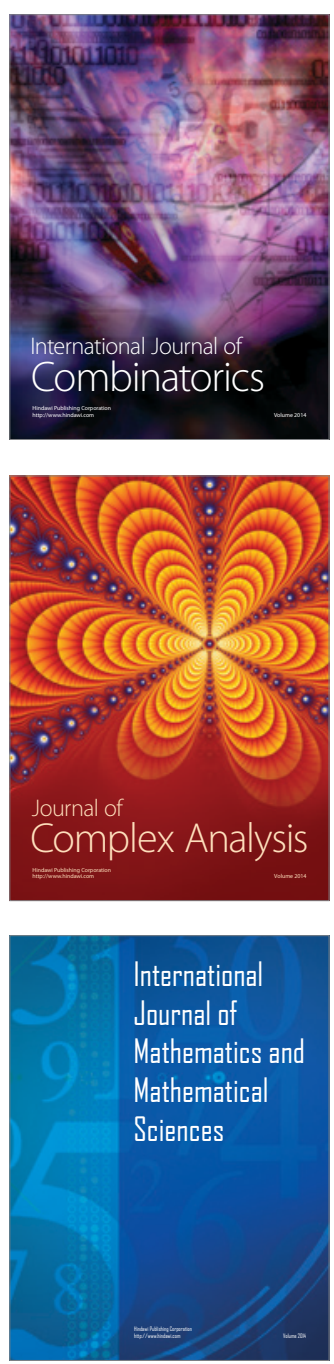
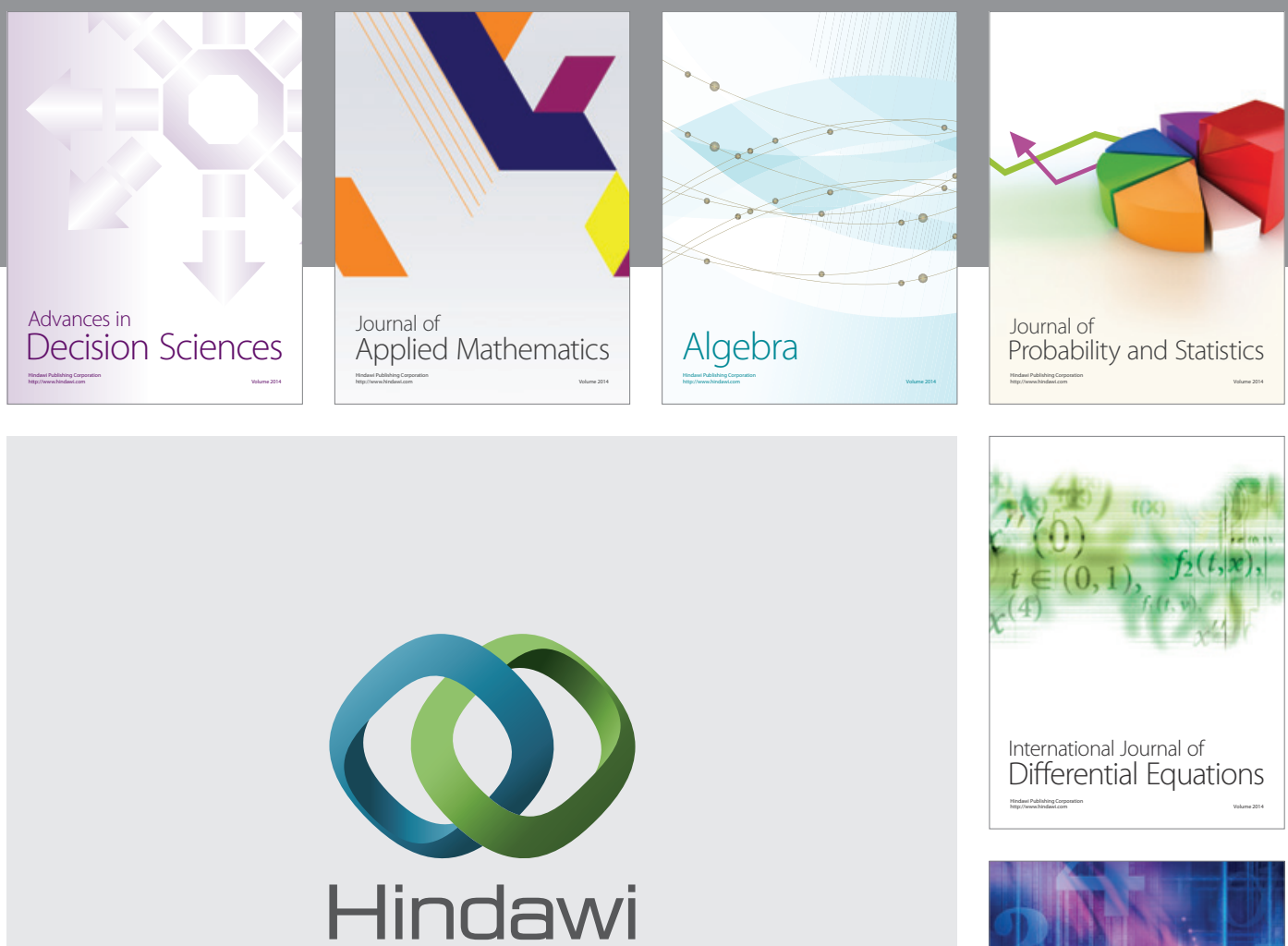

Submit your manuscripts at http://www.hindawi.com
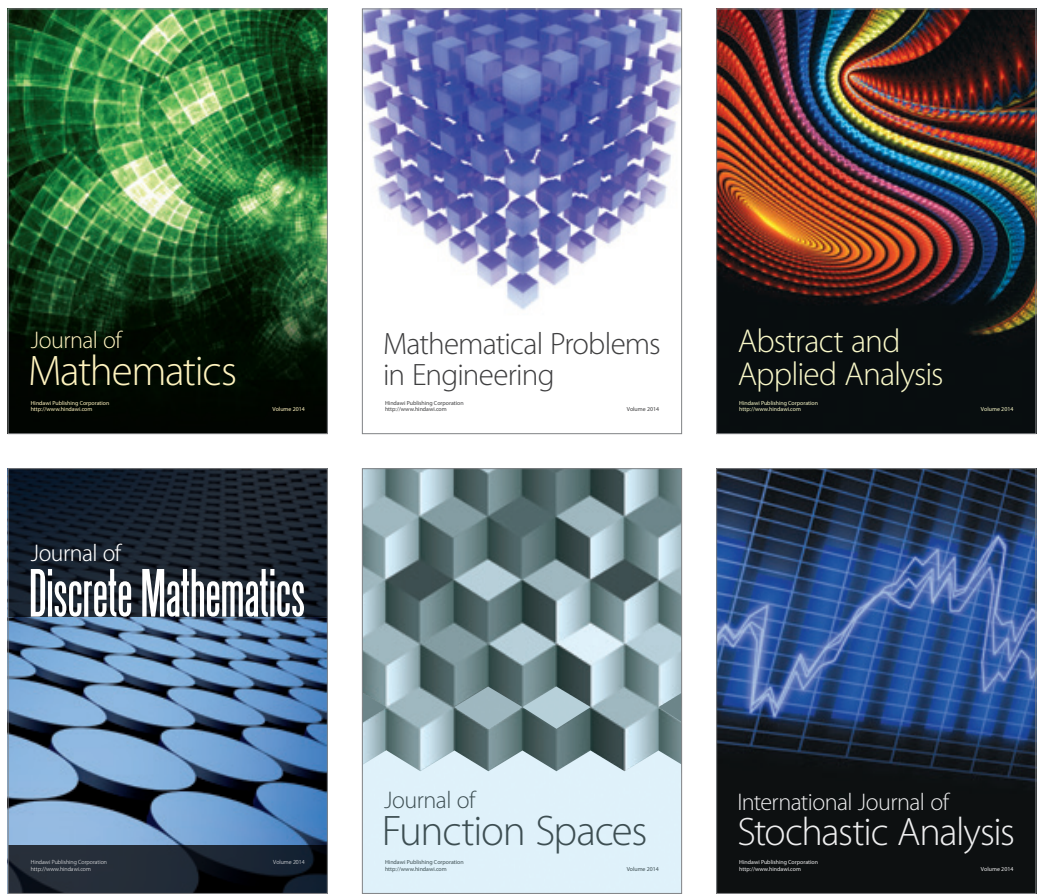

Journal of

Function Spaces

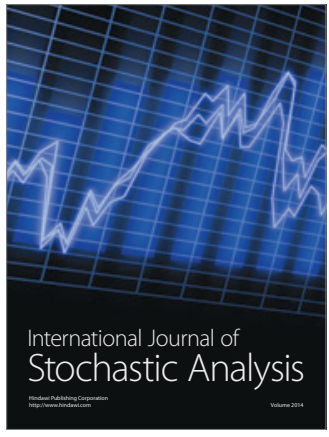

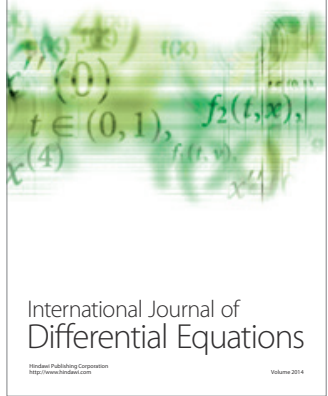
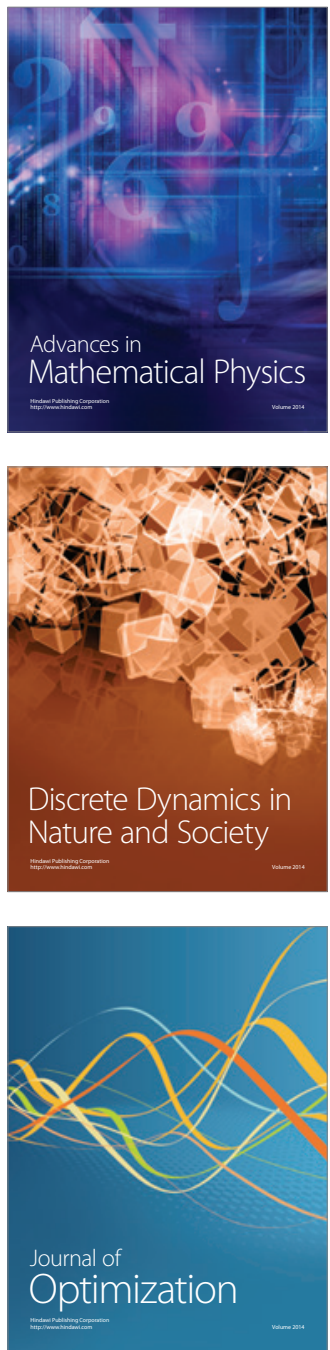